\title{
Bubble Behavior in Nucleate Boiling Experiment Aboard the Space Shuttle
}

\author{
Justin P. Koeln ${ }^{1}$, Jeffrey C. Boulware ${ }^{1}$ and Heng Ban ${ }^{2}$ \\ Utah State University, Logan, Utah, 84322
}

\begin{abstract}
Boiling dynamics in microgravity need to be better understood before heat transfer systems based on boiling mechanism can be developed for space applications. This paper presents the results of a nucleate boiling experiment aboard Space Shuttle Endeavor (STS108). The experiment utilized nickel-chromium resistance wire to boil water in microgravity, and the data was recorded with a CCD camera and six thermistors. This data was analyzed to determine the behavior of bubble formation, detachment from the heating wire, and travel in the water with effects of drag on bubble movement. Bubbles were observed to be ejected from the wire, travel through and eventually stop in the unsaturated water. The data from this experiment is in good agreement with the results of theoretical equations used to model bubble-fluid dynamics in microgravity. The primary conclusion from this experiment is that a bubble can be ejected from a heated wire in the absence of gravity, instead of the creation of a single large vapor bubble. Further conclusions from this research could be applied to the development of safe and efficient heat transfer systems for microgravity and terrestrial applications.
\end{abstract}

\section{Nomenclature}

$\begin{array}{ll}a & =\text { drag coefficient prediction constant } \\ A & =\text { bubble cross-sectional area } \\ C_{d} & =\text { coefficient of drag } \\ \mathrm{D} & =\text { bubble diameter } \\ F_{d} & =\text { drag force } \\ m & =\text { bubble mass } \\ M o & =\text { Morton number } \\ \mathrm{Re} & =\text { Reynolds number } \\ \sigma & =\text { surface tension } \\ t & =\text { time } \\ v & =\text { bubble velocity } \\ W e & =\text { Weber number } \\ x & =\text { distance from wire } \\ \rho_{l} & =\text { density of water }\end{array}$

\section{Introduction}

A S technology advances toward the development of space, safe and effective heat transfer systems using phase change mechanisms are needed for large scale power production and thermal management. Before these systems can be developed a better understanding of boiling and bubble dynamics during nucleate boiling in microgravity is needed. The absence of free convection due to buoyancy reduces the convective heat transfer on orbit, resulting in more localized heating and larger thermal gradients. This paper presents the results of a thin wire nucleate boiling experiment performed on orbit aboard Space Shuttle Endeavor (STS-108). The experiment showed that, with a sufficient heating rate, bubble ejection from the heating element is possible even without buoyancy.

\footnotetext{
${ }^{1}$ Student, Dept. of Mechanical and Aerospace Engineering, 4130 Old Main Hill, Logan, UT 84322-4130, Student Member.

${ }^{2}$ Associate Professor, Dept. of Mechanical and Aerospace Engineering, 4130 Old Main Hill, Logan, UT 843224130, Member.
} 
Based on visual and thermal data from the experiment, correlations of the path of a bubble after departing the wire were developed with numerical predictions based on drag dynamics.

\section{Background and Theory}

\section{A. Nucleate Boiling}

Different modes of boiling occur based on the difference between the excess temperature and the heat flux into the fluid. The excess temperature is the difference between the temperature of the fluid and its saturation temperature. Figure 1 shows the different boiling regimes for water at $1 \mathrm{~atm}$. As seen from the figure, nucleate boiling can be divided into two sections: isolated bubbles and jets and columns. As the fluid becomes hotter, increasing the excess temperature, nucleation sites activate and more bubbles begin to form on the surface. The low end of the nucleate boiling regime has relatively few nucleation sites producing distinct isolated bubbles. The upper end of the nucleate boiling regime has many nucleation sites in close proximity

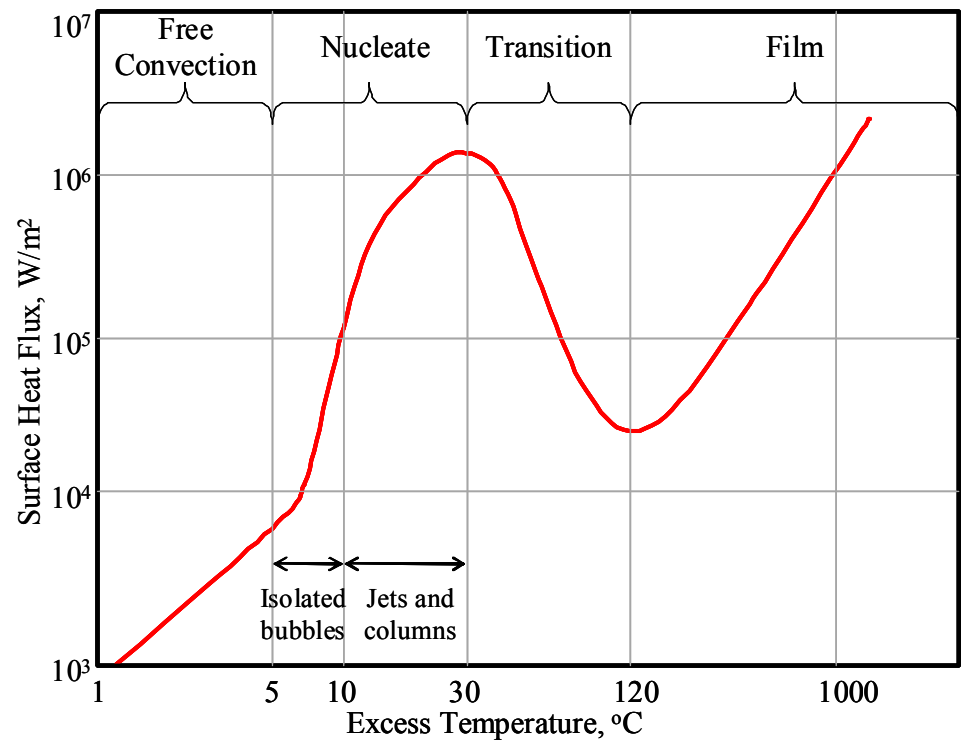

Figure 1. Boiling regimes for water at 1 atm, taken from Incropera ${ }^{1}$.

causing bubble interference and coalescence. This study observes formation and departure of isolated bubbles and therefore only deals with segment of the boiling regime where the excess temperature is between 5 and 10 degrees Celsius. Many terrestrial engineering devices take advantage of the nucleate boiling regime due to the high heat transfer rates and convection coefficients associated with small values of excess temperature. For space applications, the formation, growth, departure, and travel history of bubbles control the heat transfer coefficient of boiling heat transfer on the surface; therefore, this study intends to provide detailed description of the bubble growth, departure and travel history. There is also a significant difference between 1-g and 0-g boiling: the water in $1 \mathrm{-g}$ boiling is generally at saturation temperature, whereas in 0 -g, the lack of convection causes the water to only be at saturation temperature close to the heating surface. Away from the heating surface, the water can be significantly below saturation temperature.

\section{B. Previous Research on Nucleate Boiling in Microgravity}

Extensive research has been performed to understand the forces involved in nucleate boiling on Earth. Studies have verified theoretical calculations of inertia, buoyancy, surface tension, and drag as a bubble nucleates and travels through a fluid as well as the bubble's diameter and contact angle upon departure; however, very little nucleate boiling research has been performed on-orbit. Without the dominant force of buoyancy, bubble dynamics and heat transfer differ greatly. Much research was conducted on microgravity simulators such as NASA's KC$135 \mathrm{~A}$ and drop towers, but these methods can only simulate microgravity for a brief period of time and cannot eliminate all effects of gravity.

Nonetheless, thin-wire reduced gravity experiments on nucleate boiling have been carried out on drop towers (Siegel and Usiskin ${ }^{2}$, Tokura ${ }^{3}$, Motoya ${ }^{4}$, Sitter $^{5}, \mathrm{Zhao}^{6}$ ), parabolic flights $\left(\right.$ Straub $^{7,8,9}$, Shatto and Peterson ${ }^{10}$, Di Marco and Grassi ${ }^{11}$ ), sounding rockets (Di Marco ${ }^{12}$ ), the Space Shuttle (Steinbichler ${ }^{13}$, Hasan $^{14}$ ), and satellites $\left(\mathrm{Zhao}^{15,16}\right)$. Unfortunately, none of these experiments studied water, instead opting for refrigerants and electrical fluids. Furthermore, the studies resulted in conflicting trends for the critical heat flux and non-intuitive bubble behavior. Most experiments resulted in one large bubble wrapping the wire and causing a burnout in the wire due to lack of heat flux. Prior to the coalescence into one large bubble, lateral oscillations of smaller bubbles along the wire occurred. The current study observed the effects by heating a single straight wire and then three braided wires; 
however, no lateral oscillations occurred in either case and large bubble coalescence did not occur over the entire test length.

\section{Drag Force on a Vapor Bubble}

On the wire, the bubble undergoes an initial acceleration due to vapor accumulation and momentum overcoming surface tension attaching the bubble to the wire. Upon departure from the heating surface, the bubble decelerates due to the drag force exerted by the water. Drag, $F_{d}$, is a function of the coefficient of drag, $C_{d}$, the density of the fluid, $\rho_{l}$, the cross-sectional area, $A$, and the velocity relative to the liquid, $v$, as shown below in Equation 1.

$$
F_{d}=-\frac{1}{2} \rho_{l} v^{2} A C_{d}
$$

Without buoyancy, the force balance simply consists of the drag force and change in momentum as shown in Equation 2.

$$
F_{d}=\frac{d(m v)}{d t}
$$

Classic nucleation theory estimates the bubble mass as $11 / 16$ of the mass of the fluid which would occupy the space of the bubble. This estimation, developed by Han and Griffith ${ }^{17}$, accounts for fluid carried with the bubbles during transit. Thus, assuming negligible phase change at the bubble's surface after leaving the wire, the force balance becomes as shown in Equation 3.

$$
-\frac{1}{2} \rho_{l} v^{2}\left(\frac{\pi}{4} D^{2}\right) C_{d}=\frac{11}{16} \rho_{l}\left(\frac{1}{6} \pi D^{3}\right) \frac{d v}{d t}
$$

Simplified, the equation becomes,

$$
\frac{d v}{d t} v^{-2}=-\frac{12}{11} \frac{C_{d}}{D}
$$

For a constant drag coefficient, the integration is simple; however, as the bubble moves out towards colder water, the drag coefficient changes over time, thereby complicating the integration. For data processing, it is convenient to use the discretized velocity and displacement functions,

$$
\begin{gathered}
v_{i}=v_{i-1}-\frac{12}{11} v_{i-1}{ }^{2} \frac{C_{d, i-1}}{D} \Delta t \\
x_{i}=x_{i-1}+v_{i} \Delta t
\end{gathered}
$$

The bubble diameter remains fairly constant in the current study; however, as aforementioned, the drag coefficient varies due to the bubble moving into cold water. This is ultimately due to the temperature dependency of the viscosity of the water as shown in Figure 2. The change in viscosity effects the Reynolds number; thereby effecting the drag coefficient. Figure 3 shows the drag coefficient versus Reynolds number for a solid sphere. 


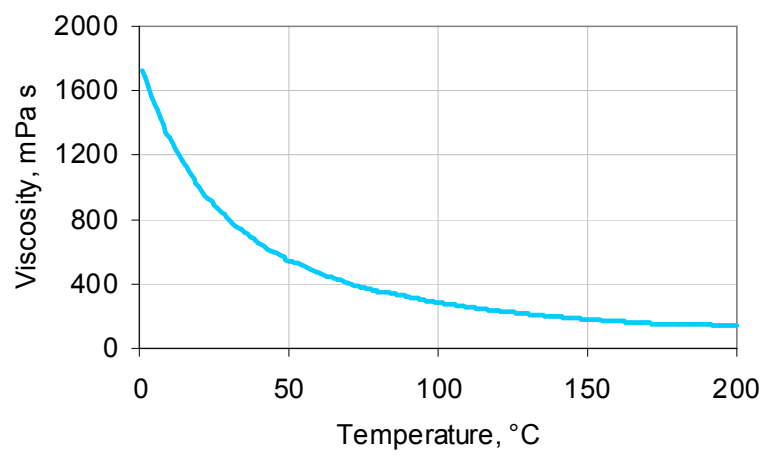

Figure 2. Viscosity of water versus temperature.

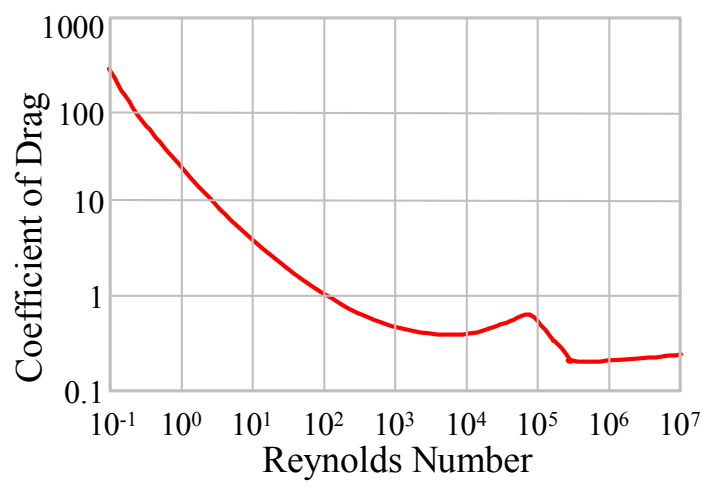

Figure 3. Drag coefficient versus Reynolds number for a solid sphere.

Several models exist which attempt to numerically predict the drag coefficient for a bubble at various Reynolds numbers. Gorring and Katz ${ }^{18}$ presented a number of correlations based on the function

$$
C_{d}=\frac{a}{\mathrm{Re}}
$$

where the constant $a$ is dependent on the flow regime. Moore's relation assumes $a=32$ and was used for this study within its limited range of Reynolds numbers. A more recent model by Kelbaliyev and Ceylan ${ }^{19}$ integrates the full regime of $0.5<\operatorname{Re}<100$ as shown below:

$$
C_{d}=\frac{16}{\operatorname{Re}}\left[1+\left(\frac{\mathrm{Re}}{1.385}\right)^{12}\right]^{1 / 55}
$$

Note that shape deformation of the bubble in this experiment can be considered negligible because it meets the relation developed by Kelbaliyev and Ceylan ${ }^{20}$

$$
\operatorname{Re} \mathrm{Mo}^{1 / 6}<7
$$

where $\mathrm{Mo}=4 / 3 * \mathrm{C}_{\mathrm{d}} * \mathrm{We}^{3} * \mathrm{Re}^{-4}$ and $\mathrm{We}=\rho_{1} * \mathrm{v}^{2} * \mathrm{D} / \sigma$. Correlations for Moore's relation and the Kelbaliyev model were compared to the experimental data.

\section{Objectives}

The research was performed with the following objectives:

1) Observe the nucleate boiling from single and braided thin wires in reduced gravity

2) Obtain position, velocity, and acceleration data from visual recording of nucleate boiling process in microgravity

3) Verify drag force equations to analytically predict the propagation of bubbles after departing the wire

4) Utilize measured temperature data to determine properties of water during boiling and obtain a thermal map of the fluid without buoyancy

\section{Test Description}

The experiment consisted of a fluid chamber containing distilled water, the heating elements, and thermistors while a video camera recorded data. The fluid chamber consisted of five Lexan walls and one Viton rubber wall to allow for expansion in the case of sub-freezing temperatures during stowage. The heating elements were two Nichrome wires, of which one was a braid of three strands and the other was a single strand. Six YSI 441107 
Teflon-encapsulated thermistors were positioned at various distances from the heating elements. A CCD camera visually recorded the boiling. The fluid chamber and schematic are shown in Figure 4 and Figure 5, respectively. The braided heating element was powered by 7 volts for 35 minutes by 40 lead X-cell batteries; unfortunately this data was not recorded real-time. After the braided wire was turned off the boiling chamber was able to cool for one hour until the straight wire was powered for 35 minutes. The single wire was powered afterwards, but the data in this study was provided by the braided wire solely. The CCD camera recorded the boiling and was digitized at 15 frames per second and 720 by 540 pixel resolution.

\section{Experimental Results}

\section{A. Temperature}

Each thermistor measured the temperature of the water once per minute throughout the experiment. Figure 6 shows the temperature

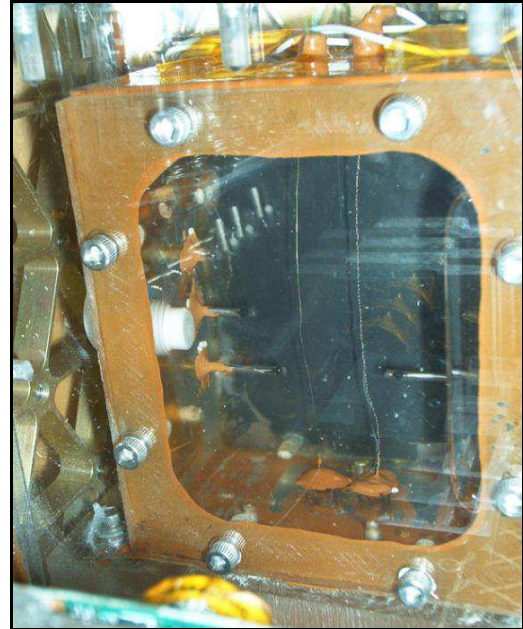

Figure 4. STS-108 experiment . readings for 4 of the 6 thermistors.

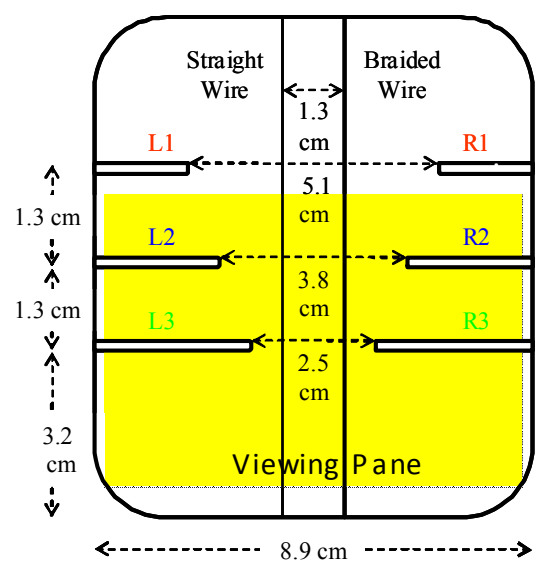

Figure 5. Chamber Schematic

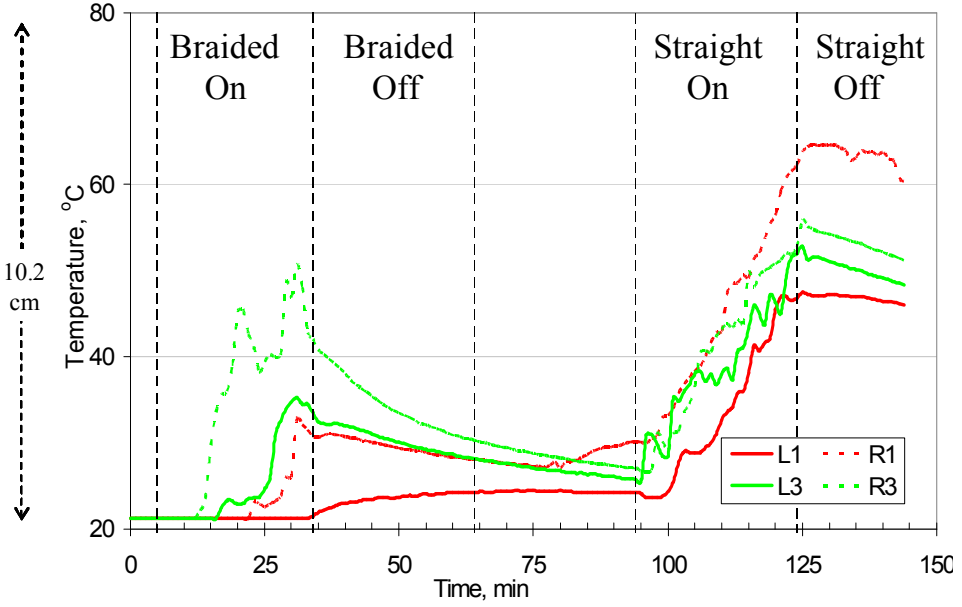

Figure 6. Thermistor readings over time.
The data from L2 and R2 after the first 25 minutes was unreasonable. These thermistors may have experienced hardware failures due to possible damage during the launch. Convection from bubbles may increase water temperature in a specific region, but the video shows the bubbles sparsed throughout the chamber evenly, not only in the regions of L2 and R2 as shown early on at 4 minutes in Figure 7. The L1, L3, R1, and R3 thermistors appear to have recorded reasonable data. The temperature of these four points did not change when bubbles first appeared on the braided wire in the first 9 minutes. At that moment, the water temperature adjacent to the wire must have reached about saturation $\left(100^{\circ} \mathrm{C}\right)$ while these four points, the closest being $12.7 \mathrm{~mm}$ from the wire, are still at about $21^{\circ} \mathrm{C}$. When more bubbles began ejecting from the wire, a convective flow of water resulted and the thermistor temperatures started to rise at around 15-35

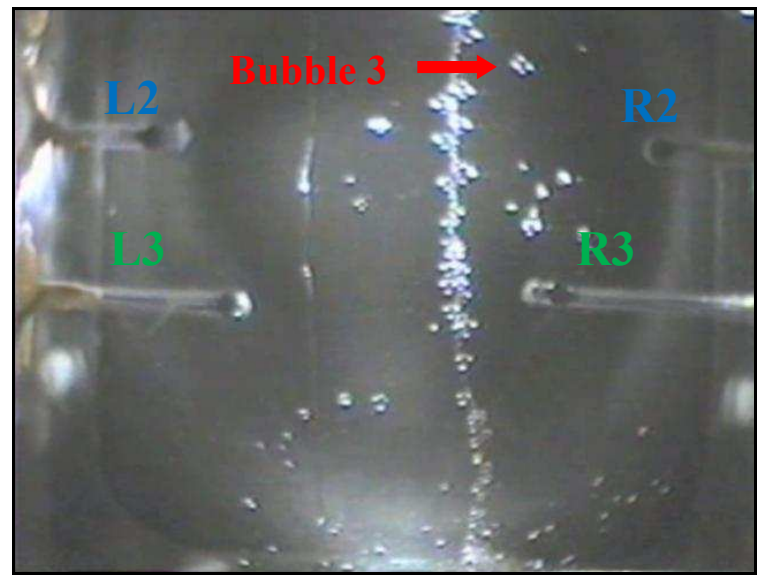

Figure 7. Photograph of nucleate boiling on a braided wire. 
minutes. When the power was turned off, temperatures decreased due to cooling through the walls. The power for the single wire was about twice that of the braided wire causing the temperature to increase almost immediately. Still, the recorded water temperature never surpassed $70^{\circ} \mathrm{C}$, about $30^{\circ} \mathrm{C}$ below saturation. This could only happen because of the absence of buoyancydriven convective flow.

\section{B. Bubble Measurement}

Within one minute of power being provided to the wire, small $(0.1-0.2 \mathrm{~mm})$ bubbles formed and detached. After two minutes, the average bubble size increased to about $1-2 \mathrm{~mm}$. After 3 to 4 minutes (when Bubbles 1, 2, 3 detached from the wire), the bubbles seemed to stop growing as rapidly. Also, at this time, many bubbles remained attached to the wire but several had propagated approximately 0.5 to $1.0 \mathrm{~mm}$ from the wire as shown in Figure 7. After 35 minutes, bubbles of varying size, including very large bubbles $(5-15 \mathrm{~mm})$, were present throughout the fluid chamber. Most of the bubbles left the wire perpendicularly but several depart at sharp angles. Most bubbles remained less than $25 \mathrm{~mm}$ from the wire but several propagated to the walls of the chamber.

Using the video, pictures were extracted at a rate of 10 frames per second for the first second after the separation of the bubble from the heating element for three bubbles. These three bubbles were chosen because they appeared to travel on the plane perpendicular to the camera. The size of the bubbles can be estimated to be about 1.5 $\mathrm{mm}, 1.5 \mathrm{~mm}$ and $2 \mathrm{~mm}$ for Bubbles 1, 2, and 3, respectively, with $\pm 10 \%$ uncertainty due to poor resolution and lighting conditions. Figure 7 shows an example of a picture created from the video file. The bubble in the upper right corner is Bubble 3.

The position of the bubble over time was obtained by finding the pixel corresponding to the center of the bubble in each picture where each pixel corresponds to approximately $0.1 \mathrm{~mm}$ physical length. Figure 8 is a graph of the position of the three bubbles over time. The time in the charts is the time relative to the bubble leaving the wire.

The velocity (Figure 9) of the bubbles was approximated using a first-order, center differencing approach for the differentiation.

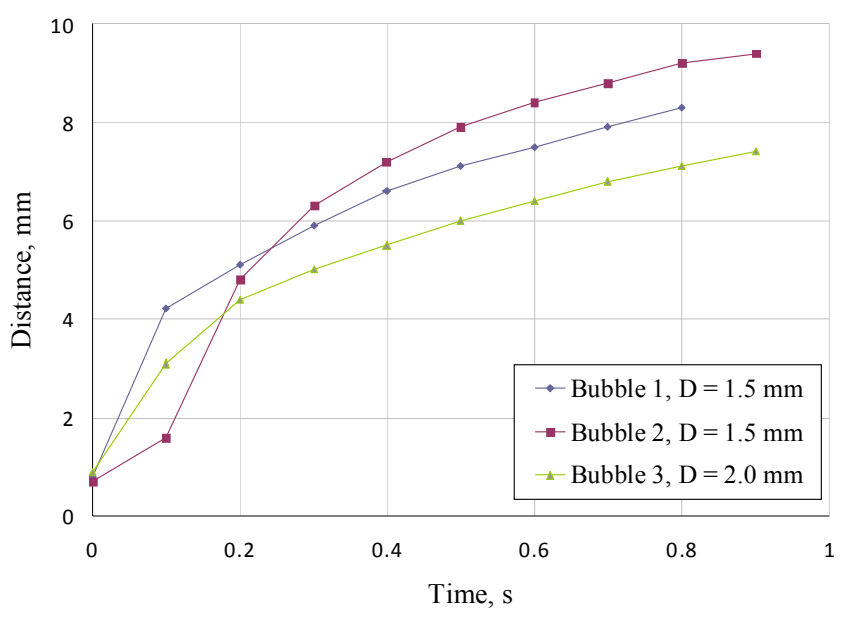

Figure 8. Bubble center displacement from wire over time.

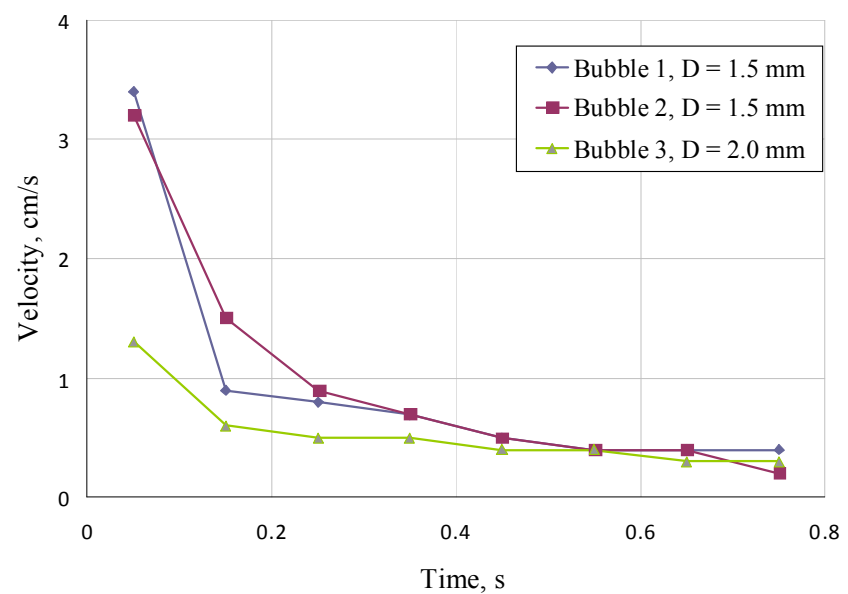

Figure 9. Bubble velocity after departure over time.

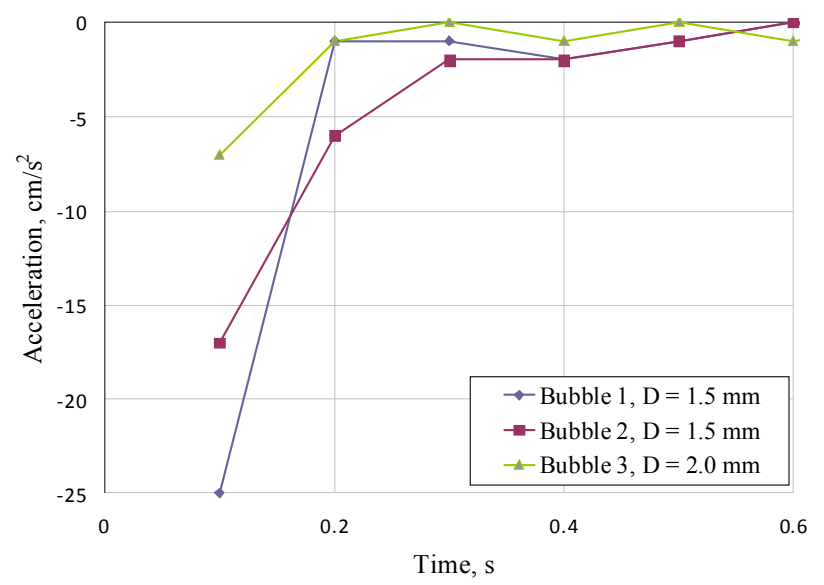

Figure 10. Bubble deceleration after departure over time due to drag. 


$$
v_{i+\frac{1}{2}}=\frac{x_{i+1}-x_{i}}{\Delta t}
$$

Due to the finite-differencing approach the number of data points decreases by one after each differentiation. The velocity of the bubble is greatest right after it breaks free of the wire then quickly reduces to zero due to drag. The acceleration (Figure 10) of the bubbles was also approximated using a first-order, center differencing, discretization approach.

$$
\left(\frac{d v}{d t}\right)_{i+\frac{1}{2}}=\frac{v_{i+1}-v_{i}}{\Delta t}
$$

\section{Analysis and Discussion}

\section{A. Model Predictions}

The predicted paths of a bubble after departing the thin wire determined using Moore's relation and the Kelbaliyev model are presented in Figure 11 Figure 13. It is evident that both models generally agree well with the experimental data, although Moore's model tends to yield more travelling distance than the Kelbaliyev model. Both prediction curves tend to plateau slightly quicker than the measured data for all three bubbles. Given the empirically found model inputs, Moore's relation initially overestimates the bubble position for approximately the first second after leaving the wire. Conversely, the Kelbaliyev model always under predicts the displacement of the bubble for all three bubbles. The prediction paths for Bubble 3 appear to fit the measured data most accurately.

\section{B. Experimental Uncertainty}

The experimental uncertainty comes mainly from three parameters, bubble size, traveling direction, and time step. Figure 14 provides insight to the effects of measurement uncertainty of bubble size on the prediction methods for Bubble 1. The dashed lines represent a change in diameter of the bubble by one pixel. The effects of the diameter of the bubble are quite significant yet due to the resolution of the video and poor lighting, bubble diameter had to be approximated to within $\pm 0.1 \mathrm{~mm}$.

The motion of the bubble was measured in only two dimensions. Movement toward or away from the camera was not taken into effect when measuring a bubble's distance from the wire

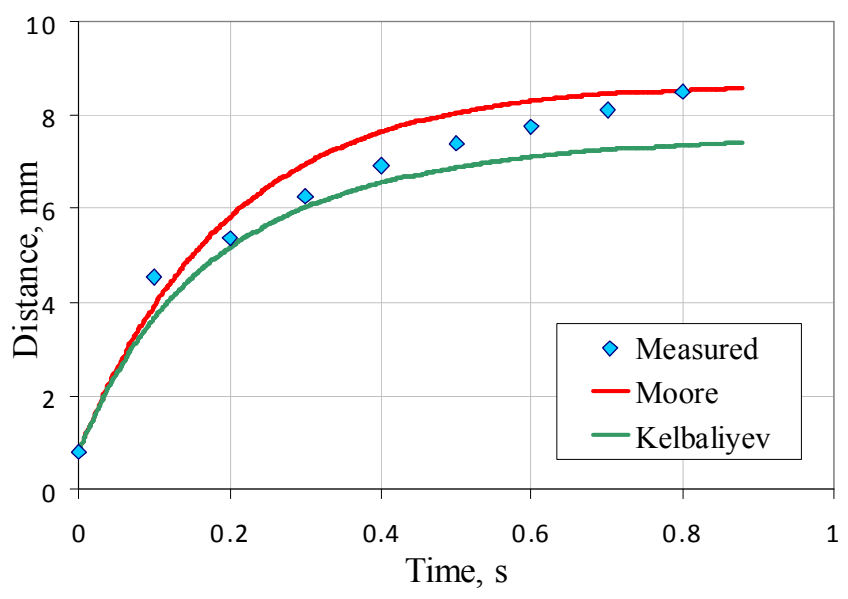

Figure 11. Measured and predicted displacement over time for Bubble 1 (diameter $=1.5 \mathrm{~mm}$ ).

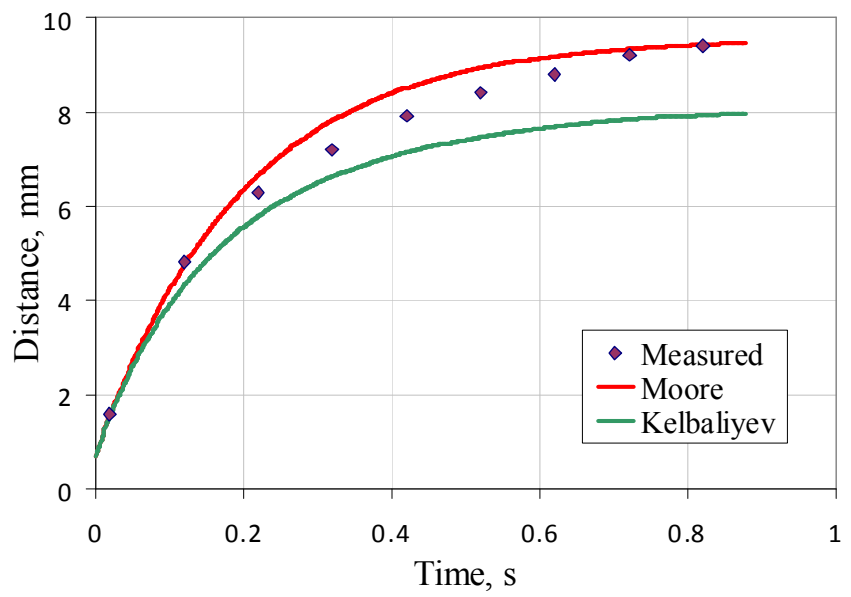

Figure 12. Measured and predicted displacement over time for Bubble 2 (diameter $=1.5 \mathrm{~mm})$.

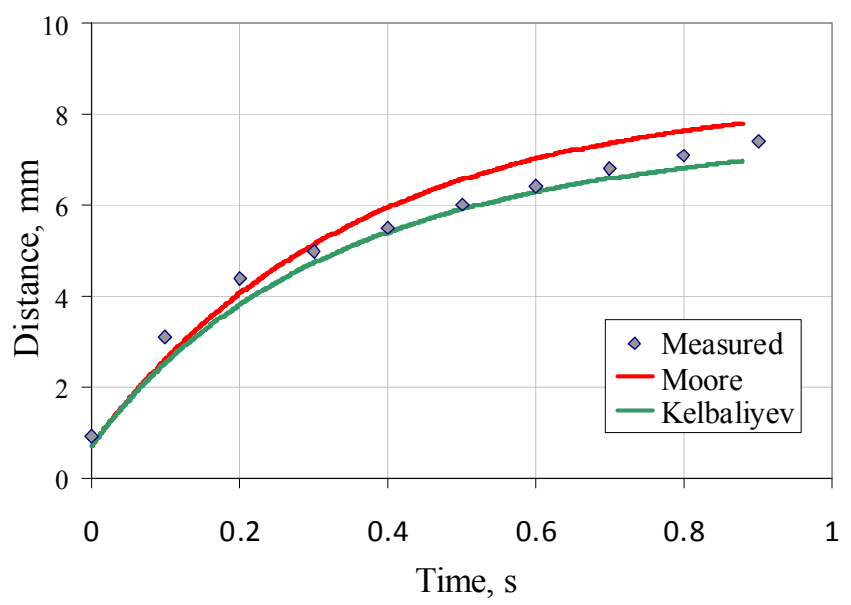

Figure 13. Measured and predicted displacement

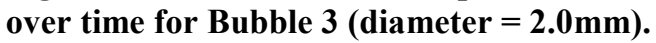


because it could not be seen. Any motion in this third dimension would increase the bubble's measured distance, velocity and acceleration. Furthermore, while the added motion to/from the camera would add to the total dynamics of the bubble, the input parameters for the numerical model were based on of what was seen. In other words, it cannot be claimed that overestimation by Moore's relation is more accurate than the Kelbaliyev model because the model inputs were determined empirically.

The effects of the discrete position measurements are most apparent when the bubble has the highest velocity as soon as it breaks free of the wire. As the time step

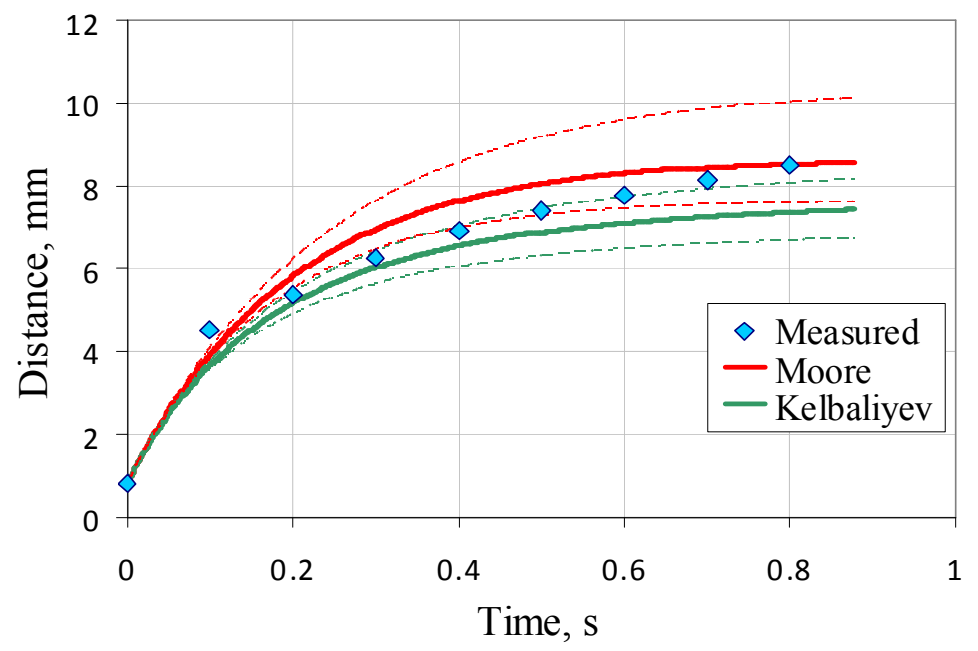

Figure 14. Effects of bubble diameter on predicted displacement. becomes smaller, the bubbles position and velocity upon departure is known more precisely. The time step was limited by digitization capabilities and frame rate of the CCD camera. With a time step of 0.1 seconds for velocities on the order of $\mathrm{cm} / \mathrm{s}$, a precise value for the initial velocity could not be determined. Thus, the initial velocity for both prediction methods was estimated to best fit the measured data.

\section{Conclusions and Recommendations}

Based on the STS-108 experiment on nucleate boiling and the correlation of the two prediction methods, certain conclusions can be drawn. Primarily, it is possible to eject a bubble from its heating surface without gravity, so long as the heating rate is sufficient. Furthermore, the drag force on a bubble after departing a thin wire can be calculated accurately using either Moore's relation or the Kelbaliyev model, but only as long as well-defined model inputs are used. In regards to the study's objectives, the following conclusions can be made:

1) A bubble can be ejected from a heated wire in the absence of gravity, most likely due to the momentum in bubble growth overcoming the surface tension force that holds the bubble to the wire

2) Moore's relation and the Kelbaliyev equation can be used to accurately model bubble drag and travel from a thin wire in zero gravity

3) Conduction-induced thermal gradients within the water without buoyancy are still affected by convection currents generated by the bubbles ejected off the heater wire, resulting in a complex temperature field.

Further experimentation should take these conclusions into consideration, as well as recommendations on specific aspects of the experimentation as follows:

1) Structural design: The distance between the wall of the fluid chamber and the heating element should be increased to minimize wall boundary effects.

2) Thermal recording: The thermistors used should be more robust and plentiful to measure the temperature of the fluid closer to the heating element to better map the thermal gradients throughout the fluid.

3) Video recording: The camera used should be of higher resolution and faster frame rate. Multiple cameras or mirrors should be used to view the boiling chamber from multiple angles in order to determine the bubbles' position in three dimensions.

4) Lighting: The fluid chamber should have multiple light sources from different angles in order to illuminate the bubbles more uniformly.

5) Power supply: The power delivered to the heating element should be decreased to reduce the number of bubbles in order to better see the formation and departure of individual bubbles and to study its effect on the bubbles' initial acceleration. 
6) Power recording: The applied voltage and current should be continuously recorded to provide insight on the power entering the system.

In summary, this study has contributed valuable knowledge on the behavior of bubbles during nucleate boiling by demonstrating that certain heating conditions can prevent the coalescence of a large solitary bubble and bubbles can be ejected from the heater wire to generate significant convection currents. This phenomenon was neither intuitive nor easily discovered. The knowledge and information learned from this study will aid the development of safe and efficient heat transfer systems for microgravity and terrestrial applications.

\section{Acknowledgments}

The USU Get Away Special (GAS) team of 2001, particularly Jeffrey Duce, is acknowledged for their effort of designing and performing the experiment. Acknowledgement is also due to all organizations who have donated funding or equipment including YSI Thermistors, ATK Thiokol, Campbell Scientific, True North Software, Ecco Computers, Shoshone-Bancock Native American High School, Space Dynamics Laboratory, and the Rocky Mountain NASA Space Grant Consortium.

A special thanks is deserved to Dr. Jan Sojka, the GAS team advisor, and Dr. J. R. Dennison for their leadership and support throughout the years. The project's synthesis is credited to Ron Cefalo and his students at Box Elder High School whose inquisitive nature led to the first attempt of the experiment on STS-091 paving the way for the current project's success.

\section{References}

${ }^{1}$ Incropera, F. P., Dewitt, D. P., Bergman, T. L., and Lavine, A. S., Fundamentals of Heat and Mass Transfer, $6^{\text {th }}$ ed., John Wiley and Sons, New Jersey, 2007, pp. 621-626.

${ }^{2}$ Siegel, R., and Usiskin, C., "Photographic Study of Boiling in Absence of Gravity," Journal of Heat Transfer, Trans. ASME, Vol. 81C, 1959, pp. 230-236.

${ }^{3}$ Tokura, I., Hanaoka, Y., Suzuki, H., Hirata, H., and Yoneta, M., "Boiling Heat Transfer on Thin Wires in a Microgravity Field," Proceedings of the ASME/JSME Thermal Eng. Conference, Vol. 4, Maui, HI, 1995, pp. 555-560.

${ }^{4}$ Motoya, D., Haze, I., and Osakabe, M., "Effect of Fouling on Nucleate Pool Boiling in Microgravity and Earth Gravity," ASME Heat Transfer Division, Vol. 364-1, 1999, pp. 303-310.

${ }^{5}$ Sitter, J.S., Snyder, T.J., Chung, J.N., and Marston, P.L., "Acoustic Field Interaction with a Boiling System Under Terrestrial Gravity and Microgravity,” Journal of the Acoustical Society of America, Vol. 104, 1998, pp. 2561-2569.

${ }^{6}$ Zhao, J.F., Wan, S.X., Liu, G., and Hu, W.R., "Experimental Study on Subcooled Pool Boiling in Microgravity Utilizing Drop Tower," Proceedings of the International Symposium on Multiphase Flow, Beijing/NMLC., Xi'an, China, 2005.

${ }^{7}$ Straub, J., "Boiling Heat Transfer and Bubble Dynamics in Microgravity," Advances in Heat Transfer, Vol. 35, 2001, pp. $57-172$.

${ }^{8}$ Straub, J., and Micko, S., "Boiling on a Wire Under Microgravity Conditions - First Results from a Space Experiment," Proceedings of Eurotherm Seminar No. 48, Paderbom, Germany, 1996.

${ }^{9}$ Straub, J., Zell, M., and Vogel, B., "Boiling Under Microgravity Conditions," Proceedings of the 1st European Symposium of Fluids in Space, ESA SP-353, Ajaccio, France, 1992.

${ }^{10}$ Shatto, D.P., and Peterson, G.P., "Pool Boiling Critical Heat Flux in Reduced Gravity,". Journal of Heat Transfer, Trans. ASME, Vol. 121, 1999, pp. 865-873.

${ }^{11}$ Di Marco, P., and Grassi, W., "Motivation and Results of a Long-Term Research on Pool Boiling Heat Transfer in Low Gravity," International Journal of Thermal Sciences, Vol. 41, 2002, pp. 567-585.

${ }^{12}$ Di Marco, P., Grassi, W., and Trentavizi, F., "Pool Film Boiling Experiments on a Wire in Low Gravity: Preliminary Results," Microgravity Transport Processes in Fluid, Thermal, Biological \& Material Sciences, edited by S. S. Sadhal, Vol . 26, Annals of the New York Academy of Sciences, 2001. 
${ }^{13}$ Steinbichler, M., Micko, S., and Straub, J., "Nucleate Boiling Heat Transfer on Small Hemispherical Heaters and a Wire Under Microgravity," Proceedings of the $11^{\text {th }}$ International Heat Transfer Conference, Vol. 2, Kyongju, Korea, 1998, pp. 539544.

${ }^{14}$ Hasan, M. M., Lin, C. S., Knoll, R. H., Bentz, M. D., and Meserole, J.S., "Nucleate Pool Boiling in the Long Duration Low Gravity Environment of the Space Shuttle," NASA TM-105973, 1993.

${ }^{15}$ Zhao, J. F., Liu, G., Wan, S. X., and Yan, N., "Bubble Dynamics in Nucleate Pool Boiling on Thin Wires in Microgravity," Microgravity Science and Technology, Vol. 20, 2008, pp. 81-89.

${ }^{16}$ Zhao, J. F., Wan, S. X., Liu, G., Yan, N. and Hu, W. R., "Subcooled Pool Boiling on Thin Wire in Microgravity," Acta Astronautica, Vol. 64, 2009, pp. 188-194.

${ }^{17}$ Han, C., and Griffith, P., "The Mechanism of Heat Transfer in Nucleate Pool Boiling," International Journal of Heat and Mass Transfer, Vol. 8, 1965, pp. 887-904.

${ }^{18}$ Gorring, R. L., and Katz, D. L., "Bubble Rise in a Packed Bed Saturated with Liquids", American Institute of Chemical Engineers Journal, Vol. 8, No. 1, 1962, pp. 123-126.

${ }^{19}$ Kelbaliyev, G., and Ceylan, K., "Development of New Empirical Equations for Estimation of Drag Coefficient, Shape Deformation, and Rising Velocity of Gas Bubbles or Liquid Drops," Chem. Eng. Comm., Vol. 194, 2007, pp. $1623-1637$.

${ }^{20}$ Kelbaliyev, G., and Ceylan, K., "Estimation of the Minimum Stable Drop Sizes, Break-up Frequencies, and Size Distributions in Turbulent Dispersions," Journal of Dispersion Science and Technology, Vol. 26, 2005, pp. 487-494. 